\title{
Tracheal stenosis
}

\author{
Abdurahim Aloud MD
}

62-year-old woman, lifelong nonsmoker, with comorbid conditions of myelodysplastic syndrome, transfusion dependent anemia, prior gastrointestinal bleeding, and a recent hospitalization for severe anemia and hypotension treated with multiple blood transfusions, was admitted to the hospital after she presented with worsening shortness of breath, unremitting dry cough for three weeks, and right-sided chest pain. She slept in a sitting position, stating that she suffocates and constantly coughs when she sleeps flat. She was treated as an outpatient with prednisone and Symbicort $^{\circledR}$ without improvement. She denied having fever, hemoptysis, and other symptoms. On physical examination she was awake, alert, and oriented. She had bilateral crackles on lung auscultation and bilateral pitting lower extremity edema. There were reported episodes of stridor by the nursing staff, but she did not have any stridor during the physical examination.

A computed tomography scan of the thorax showed a large loculated pleural effusion on the right side and severe narrowing of the trachea at the cervicothoracic junction and at the carina, with symmetrical paratracheal soft tissue thickening (Figure 1). Laboratory work up showed an elevated BNP, bilirubin, and alkaline phosphatase. Pleural fluid was a neutrophilic exudate with negative pleural fluid cultures.

Bronchoscopy showed circumferential narrowing of the trachea $8 \mathrm{~cm}$ above the carina; the respiratory mucosa was friable and bled easily (Figure 2). She had tracheal dilation and transbronchial aspirates of the thick paratracheal tissue. Stent placement was not successful. A repeat bronchoscopy was successful in placing a stent in the left main stem bronchus.

Corresponding author: Abdurahim Aloud

Contact Information: Abdurahim.aloud@ttuhsc.edu DOI: $10.12746 /$ swrccc.v7i28.548
Tracheal biopsies showed squamous metaplasia with no evidence of malignancy or other pathological findings. Mediastinal lymph node biopsies by endobronchial ultrasound showed normal lymphoid cells with no evidence of malignancy.

The patient went into acute respiratory failure requiring supplemental oxygen by high flow nasal cannula and developed hypotension. A repeat transthoracic ultrasound showed moderate pericardial

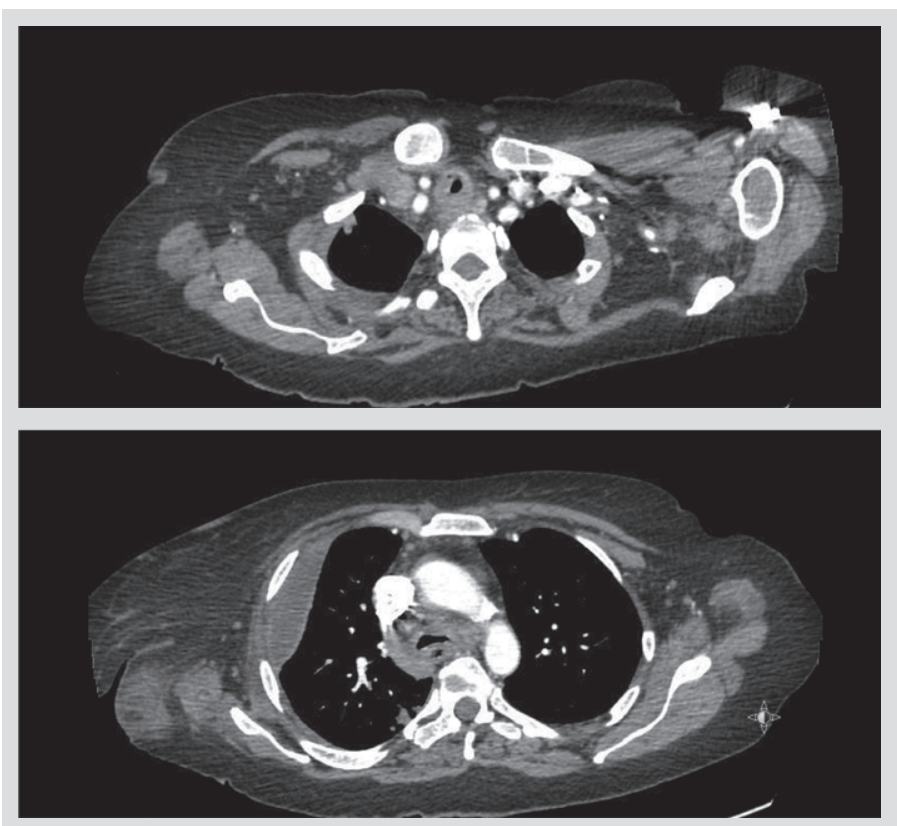

Figure 1. Severe narrowing of the trachea at the cervicothoracic junction and at the carina. There is also an inflammatory process with thickening of the wall of the trachea, especially in the region of the carina. On other images not shown, there are large loculated pleural effusions on the right side of the chest inferiorly, laterally, and in the major fissure with secondary atelectatic changes and areas of possible parenchymal scarring in the left upper lobe posteriorly. 


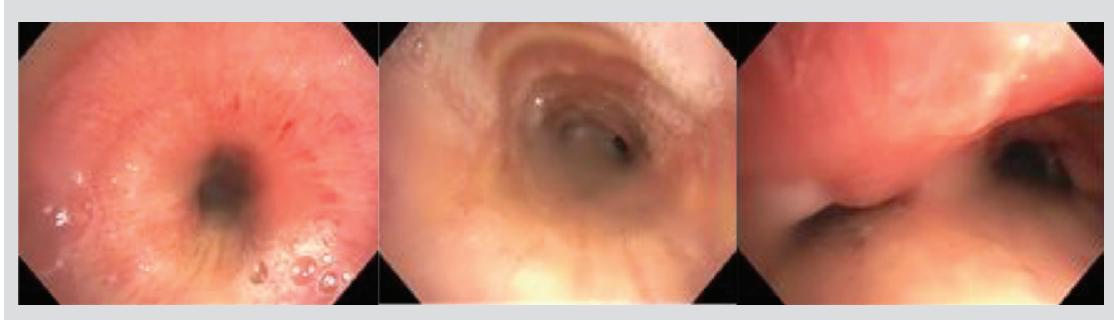

Figure 2. Bronchoscopy images showing circumferential narrowing of the tracheal lumen. effusion without evidence of tamponade and severe pulmonary hypertension. The pericardial effusion was managed as tamponade due to the hypotension and worsening shortness of breath. A pericardial window was done, and $75 \mathrm{cc}$ of pericardial fluid was drained. Postoperatively the patient remained on mechanical ventilation, became hypotensive, required 2 vasopressors, developed acute kidney injury with metabolic acidosis, and was started on continuous renal replacement therapy. She had a cardiac arrest and failed resuscitation.

\section{Discussion}

Acquired tracheal stenosis often results from trauma (mostly iatrogenic from endotracheal intubation), neoplasm, infection, vasculitis, or an inflammatory, infiltrative process. Idiopathic stenosis is a rare entity and represents a diagnosis of exclusion.

Tracheal stenosis is classified morphologically into: 1) Circumferential or concentric stenosis, which is usually caused by cuff related injury from endotracheal or tracheostomy tubes, or autoimmune disorders; 2) Triangular stenosis, which is usually caused by tracheal injury after tracheostomy, or chondritis; 3) Eccentric stenosis, which often occurs after intubation or thermal injury (inappropriate use of laser or electrocautery); 4) Complex stenosis, which happens most commonly after intubation or tracheostomy with cartilaginous injury; and 5) Simple stenosis, which is mucosal ischemia following endotracheal intubation with no associated chondritis.

This case had severe smooth circumferential mass encasing the trachea with symmetrical narrowing of the trachea at the cervicothoracic junction and the carina and thickening of the tracheal wall. This presentation is very unusual, and we were not able to find similar cases in the published literature. Biopsies were done twice and were not diagnostic.

The tracheal wall thickening suggests an inflammatory or infiltrative process. She had loculated right sided pleural effusion, and the pleural fluid was exudative with prominent neutrophils and negative cultures. This could be related to the tracheal pathology. She also had a pericardial effusion, which suggests an inflammatory process which could be autoimmune connective tissue disease or an infection. Cultures were negative; the autoimmune work up was negative. It is unclear if it is related to the tracheal pathology.

Keywords: tracheal stenosis, concentric narrowing

Article citation: Aloud A. Tracheal stenosis. The Southwest Respiratory and Critical Care Chronicles 2019;7(28):57-58

From: Department of Internal Medicine, Texas Tech University Health Sciences Center, Lubbock, Texas

Submitted: $1 / 1 / 2019$

Accepted: 4/5/2019

Reviewer: Kenneth Nugent MD

Conflicts of interest: none

This work is licensed under a Creative Commons Attribution-ShareAlike 4.0 International License.

\section{REFERENCE}

1. Miller R, Murgu S. Evaluation and Classifications of Laryngotracheal Stenosis. Revista Americana de Medicina Respiratoria [en linea] 2014, 14 (Diciembre): $<$ http://www.redalyc. org/articulo.oa? $\mathrm{id}=382138400002>$ 